\title{
Hybrid Colloids Produced by Sequential Capillarity-assisted Particle Assembly: A New Path for Complex Microparticles
}

\author{
Songbo Ni ${ }^{\mathrm{ab}}$, Ivo Buttinonia ${ }^{\mathrm{a}}$, Heiko Wolf ${ }^{\mathrm{b}}$, and Lucio Isa ${ }^{\star a}$
}

\begin{abstract}
Colloidal particles have long been under the spotlight of a very diverse research community, given their ubiquitous presence in a broad class of materials and processes, and their pivotal role as model systems. More recently, intense efforts have been devoted to the development of micro- and nanoparticles combining multiple materials in objects with a controlled architecture, hence introducing multiple functionalities and a prescribed symmetry for interactions. These particles are often called hybrid colloids or colloidal molecules, given the analogy with classical molecules presenting well defined structures and chemical compositions. Here, we review the recent progress made in our group to fabricate a broad library of hybrid colloids exploiting a novel assembly route, which uses capillary forces at the moving edge of an evaporating droplet for the sequential composition of colloidal clusters, whose geometry and chemistry can be independently programmed.
\end{abstract}

Keywords: Active colloids · Colloidal molecules · Directed assembly $\cdot$ Hybrid colloids

\section{Introduction}

Colloidal particles (micro- and nanoparticles with a size between a few $\mathrm{nm}$ and a few $\mu \mathrm{m}$ ) are an essential component of a vast plethora of naturally occurring and man-made materials and processes. In addition to their practical relevance, colloids are also successfully used to mimic and model materials at the atomic or molecular scale. ${ }^{[1,2]}$ The idea of 'colloids as big atoms' was formalized by Peter Pusey in the 1990 s, in relation to their pivotal role in elucidating open questions on the crystallization and glass transition of hardsphere systems. ${ }^{[3]}$ This concept, however, dates all the way back to Perrin's experiments in the early 1900s, who used the sedimentation of monodisperse colloids to measure Avogadro's number, and, by doing so, essentially proved the atomic nature of matter. ${ }^{[4,5]}$ The benefits of using colloidal analogues lie in the fact that the structure, dynamics and response of such systems appear on length, time and energy scales easily accessible in the lab and for which real-space observation is often possible; macroscopic material properties and structure can therefore be directly connected to

\footnotetext{
${ }^{\star}$ Correspondence: Dr. L. Isa ${ }^{a}$

E-mail: lucio.isa@mat.ethz.ch

aLaboratory for Interfaces, Soft Matter, and Assembly

Department of Materials, ETH Zurich

Vladimir-Prelog-Weg 5, CH-8093 Zurich

bIBM Research - Zurich

Säumerstrasse 4

$\mathrm{CH}-8803$ Rüschlikon
}

the behavior and positions of the "single atoms'. For decades, colloidal particles have helped shed light on a variety of fundamental phenomena, including friction, ${ }^{[6]}$ phonon propagation, ${ }^{[7]}$ phase transitions, ${ }^{[8]} \mathrm{dy}$ namical arrest, ${ }^{[9,10]}$ capillary phenomena ${ }^{[11]}$ and crystallization in ionic systems. ${ }^{[12]}$ In all of these instances, spherical particles interacting with isotropic, repulsive or attractive potentials were used.

More recently, the scope of using colloids as model systems has become significantly wider, ambitiously aiming to mimic nature's complexity. The first step in this direction requires the capability of breaking the isotropy of the interactions by introducing directionality in the particles. The simplest way of doing so is to produce shape-asymmetric particles, ${ }^{[13]}$ but the formation of complex systems in nature often additionally requires specificity of directional interactions. ${ }^{[14]}$ In 1991, during his visionary Nobel lecture, de Gennes suggested the use of novel 'building blocks' equipped with surface 'patches' that exhibit heterogeneous properties and interact specifically with other patches (e.g. A $\leftrightarrow \mathrm{B}$ interactions, but no $\mathrm{A} \leftrightarrow \mathrm{A}$ or $\mathrm{B} \leftrightarrow \mathrm{B}$ interactions). The number of patches and their symmetry hereby encode the symmetry of the structure of the final material. Hence, significant efforts have been devoted in going from 'colloidal atoms' to 'colloidal molecules', $[15,16]$ i.e. particles with prescribed geometries and chemical composition, also often called hybrid colloids.

Examples include particles or particle clusters with shapes (and interaction potentials) that resemble those of simple molecules, ${ }^{[17-19]}$ and even clusters with internal degrees of freedom that lead to different 'isotopes' of the same species. ${ }^{[20]} \mathrm{Bi}$ functional and tri-functional patchy microspheres have been assembled into a variety of structures, including reconfigurable clusters, ${ }^{[21]}$ chains, ${ }^{[22]}$ two-dimensional crystals $^{[23]}$ and open crystalline lattices. ${ }^{24]}$ Recently, DNA-coated particles introducing specific interaction symmetries have even been used to assemble large-scale crystalline structures with no atomic analogues. ${ }^{[25]}$ Even more complex structures arise when surface heterogeneity is combined with non-spherical particle symmetry. Examples include tubular ${ }^{[26]}$ and fiberlike structures ${ }^{[27]}$ obtained with ellipsoids, polymer/copolymer-like chains achieved by linking rod-like particles ${ }^{[28,29]}$ or soft multi-patchy nanospheres. ${ }^{[30]}$

Initially driven by theoretical studies and numerical simulations aimed at identifying minimal pathways to achieve complex material assembly, ${ }^{[31]}$ the experimental realization of multi-functional colloids has also recently seen an upsurge. Considering only the case of (sub)micrometer particles, existing fabrication routes have been recently reviewed. [32-34] These include glancing-angle metal deposition, ${ }^{[35]} \mathrm{mi}^{-}$ crofluidics, ${ }^{[36]}$ emulsion-templated surface functionalization, ${ }^{[37]}$ eccentric encapsulation, [38] swelling/de-swelling, ${ }^{[17,39,40]}$ and seeded bulk polymerization. ${ }^{[41,42]}$ Each of these methods has distinctive advantages in controlling the particle yield or the size, number and symmetry of the surface patches, but they all ultimately present limitations in terms of the achievable combinations of particle shape, material and functionality. For instance, mostly 
spherical patchy particles have been so far produced using glancing metal deposition, ${ }^{[43]}$ but the patch symmetry, size and number cannot be independently chosen and are set by the metal-deposition angle and the limited choice of shadowing effects. Microfluidics can be used to produce multi-phasic particles using a large range of materials; ${ }^{[36,44]}$ however, even though the particle shape can be partly influenced by the synthesis conditions, ${ }^{[45]}$ interfacial tension of the used fluids affects the final particle shape, restricting the available geometries. In order to produce larger quantities of patchy particles, bulk methods have also been developed. Amongst them, well-defined particle clusters with regular geometries can be formed inside emulsion droplets, which are subsequently shrunk and polymerized; particle protrusions from the surface of such polymerized droplets constitute the patches. ${ }^{[41]}$ Although particles with a specific number of patches can be fractionated using centrifugation to obtain colloidal molecules with symmetries resembling the ones of simple orbitals, ${ }^{[18]}$ the patch symmetry is determined by the number of particles in the cluster and each patch has the same chemical properties.

In addition to the behavior of 'passive' (Brownian) patchy particles, colloids with heterogeneous surfaces and chemical compositions have also become models for active or living matter. In contrast to systems solely and passively subjected to thermal fluctuations, active materials are able to take energy up from the environment and convert it into net displacements. Often inspired by biological organisms, including bacteria and micrometric cells, ${ }^{[46]}$ artificial analogues, also known as active colloids, have been the subject of intense recent studies. ${ }^{[47,48]}$ In particular, compositional and geometrical asymmetry can be used to trigger the formation of local asymmetric gradients around the particles, either from chemical fuel present in the environment ${ }^{[49-51]}$ or under the action of external fields (e.g. light, ${ }^{[52]}$ magnetic ${ }^{[53-55]}$ or electric fields $\left.{ }^{[56,57]}\right)$. Active particles move in randomly-oriented straight (persistent) segments, where the direction and the magnitude of the propulsion velocity are directly given by the orientation and the strength of the surrounding gradient, respectively. Being able to control the nature of the particle asymmetry in hybrid colloids becomes therefore a very powerful tool to control the properties of the active motion of micro- and nanoscale objects.

The route that we have chosen to overcome the current bottlenecks in the fabrication of fully programmable hybrid colloids is to decouple the mechanisms that define the geometrical shape of the final particles from the ones defining their composition. In order to do so, we make use of an assembly strategy where capillary forces at the evaporating edge of a moving droplet are used to direct the assembly of micron-sized colloids into predefined sites on patterned substrates. The geometry of the sites defines the shape of our hybrid colloids, while their composition can be independently controlled by sequentially filling the pattern during successive deposition steps. The rest of this article will describe the working principles of our technique and present an extract of the range of hybrid colloids that we have been able to fabricate, showcasing the possibilities for the realization of versatile active microparticles.

\section{Sequential Capillarity-assisted Particle Assembly (sCAPA) as a Tool for Programmable Hybrid Colloids}

Our assembly route is based on a 'controlled version' of the well-known coffeering effect. ${ }^{[58,59]}$ This is a pattern-formation mechanism by which an evaporating droplet containing solid particles leaves behind a ring-like stain on a surface after complete removal of the liquid. Two main ingredients are essential in this process. First, the edge of the droplet, also known as the three-phase contact line, has to be pinned on the surface to prevent its perimeter from shrinking during liquid evaporation. Second, the evaporation rate of the droplet close to the contact line is faster than on the top, leading to a net liquid flux towards the pinned meniscus. This net flux is able to transport the suspended particles towards the immobile contact line, in turn causing the accumulation of solid material at the droplet's edge. This process leads to the formation of the stain left on the surface, as shown in Fig. 1a.

The same fundamental principles apply to capillarity-assisted particle assembly or CAPA. ${ }^{[61,62]}$ The main differences between CAPA, as a controlled particle assembly method, and the simple creation of a coffee stain are: i) The evaporating droplet does not stay pinned on a substrate, but it is dragged over it at a controlled velocity and temperature. ii) The substrate presents topographical features, typically in the form of wells or traps, that define the deposition sites for the particles. The traps are lithographically fabricated on silicon masters, which are then replicated into polydimethylsiloxane (PDMS) substrates for the depositions (see Fig. 1b). The moving droplet's meniscus pins in correspondence of the traps and the resulting capillary forces guide the particle assembly (see Fig. 1c). A necessary element for successful CAPA is the evaporation-driven creation of a dense region of particles at the moving meniscus. This region, called the accumulation zone (AZ), is analogous to the edge of the coffee ring and constitutes the source of particles that are deposited in the traps. The size and the density of the accumulation zone can be tuned by adjusting the temperature, i.e. the evaporation rate relative to the speed of the moving droplet. Additional properties can be influenced by changing the droplets' surface tension and wetting using surfactants. ${ }^{[63]}$ By carrying out confocal microscopy studies, we have demonstrated that as soon as the dense AZ moves over a trap, the latter is completely filled by particles (see Fig. 1d).[60] In fact, in this dense region, particles tend to maximize entropy by occupying all the available space, including the volume of the traps. As the meniscus moves over the
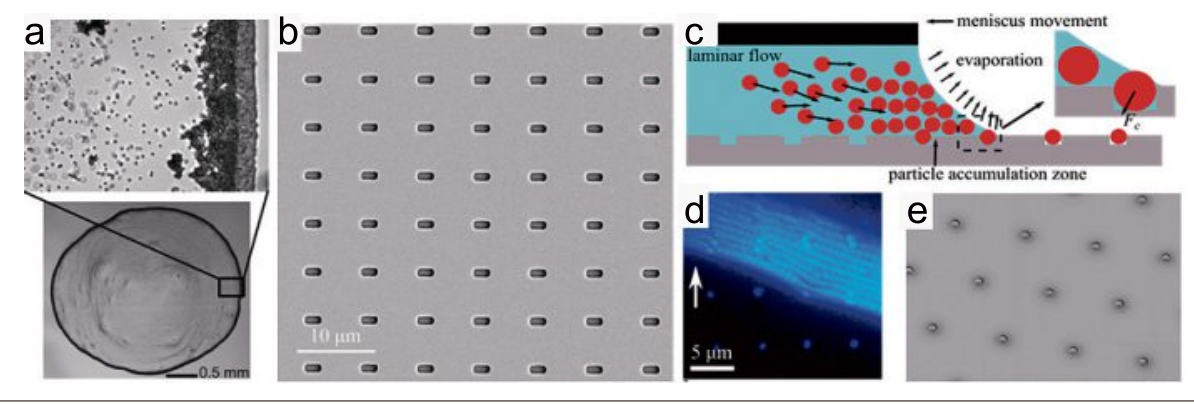

Fig. 1. a) Microscopic insight on the coffee-ring effect. Microparticles are transported toward and accumulate at the droplet's edge, causing the characteristic pattern. ${ }^{[59]}$ Adapted and reproduced by permission from Macmillan Publishers Ltd: Nature, Copyright 2016. b) PDMS template for sCAPA. The lateral trap dimensions are $1.2 \pm 0.2 \mu \mathrm{m}$ by $2.2 \pm 0.2 \mu \mathrm{m} .{ }^{[60]}$ Adapted and reproduced by permission of The Royal Society of Chemistry. c) Schematic of the working principle of capillary assembly. ${ }^{[60]}$ Adapted and reproduced by permission of The Royal Society of Chemistry. d) Confocal microscopy image of the densely packed accumulation zone at the moving meniscus during SCAPA. The white arrow indicates the direction of motion of the droplet. Individual particles are deposited in the traps, which are fully occupied by two particles in the accumulation zone (brighter particle pairs). ${ }^{[60]}$ Adapted and reproduced by permission of The Royal Society of Chemistry. e) SEM image of the successful CAPA deposition of $1 \mu \mathrm{m}$-diameter particles in $1.4 \mu \mathrm{m}$-diameter holes (depth $900 \mathrm{~nm}$ ). 
traps, the particles experience a downward capillary force perpendicular to the meniscus itself. This capillary force presses the particles into the trap and keeps them there as the meniscus depins and moves over to the next trap. For the case of traps tailored to contain only one particle, a very high efficiency of depositing individual particles can be achieved (see Fig. 1e).

If the traps are large enough to accommodate more than one particle, the situation becomes more complex and depends on several additional factors. Our experimental observations indicate that, for traps having a depth comparable to the particle diameter, the mechanisms described above for individual particles remain valid, and all traps are completely filled after one passage of the meniscus (see Fig. 2a). However, the deposition conditions can be tailored in order to guarantee the deposition of single particles only, even if the lateral dimensions of the trap would allow to host more. Confocal analysis has in fact shown that, as the meniscus interacts with the first particle in the trap, this particle is pushed towards the back and can expel other particles already present there. ${ }^{[60]}$ Consequently, only one particle is left in the trap as the meniscus depins and moves away. A more detailed description of the mechanism can be found in ref. [60]. Here, we only briefly mention that, for depths of the traps in the range of the particle radius and for appropriate values of the surface tension, the selective deposition of one particle per trap, can be successfully carried out (see Fig. 2b). This finding has crucial consequences for the fabrication of programmable hybrid colloids.

A controlled way to achieve partial filling of the traps leaves opens in fact the opportunity to back-fill the remaining available space by other particles, in a second deposition step. This idea only works if the particles assembled in the first step remain in place during the second assembly step, when exposed again to an aqueous droplet. Fig. 2c shows a clear example of this fact; here, $1200 \times 2200 \times 510 \mathrm{~nm}^{3}$ rectangular traps selectively filled by single blue $1 \mu \mathrm{m}$ polystyrene particles in a first step, are backfilled in a second capillary deposition step by analogous red particles. The micrograph shows a snapshot of the meniscus and the AZ moving over the template. The process is sketched in Fig. 2d. After the two CAPA steps, the traps are effectively filled by two different particles in contact, enabling us to realize heterogeneous dimers (see Fig. 2e). The fact that the first particles stay in the trap during exposure to the second droplet is a consequence of the increased adhesion between the colloids and the PDMS templates after drying of the first droplet. We call this process sequential capillarity-assisted particle

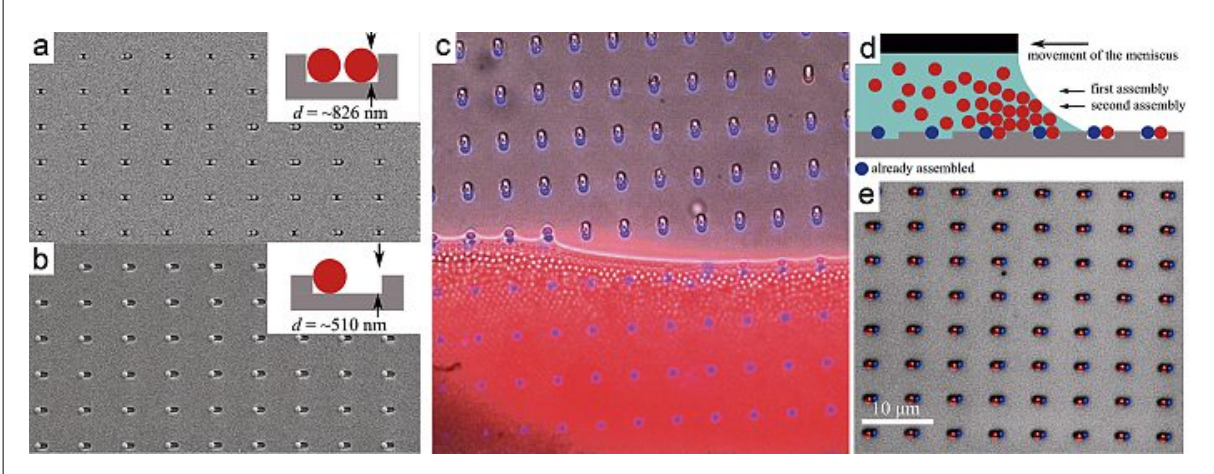

Fig. 2. a) SEM image of rectangular traps $(1.2 \pm 0.2 \mu \mathrm{m}$ by $2.2 \pm 0.2 \mu \mathrm{m})$ with a depth comparable to the particle diameter, which are completely filled by colloids ( $1 \mu \mathrm{m}$, polystyrene) after CAPA. ${ }^{[60]}$ b) SEM image of rectangular traps with a depth comparable to the particle radius, where only one particle is selectively deposited with very high yield. ${ }^{[60]} \mathrm{c}$ ) Micrograph showing the second step of sCAPA where red $1 \mu \mathrm{m}$ polystyrene particles are used to backfill the empty spaces in rectangular traps after the selective deposition of only one blue $1 \mu \mathrm{m}$ polystyrene particle per trap in the first sCAPA step. Crucially, the blue particles stay in place during the second assembly step due to adhesion with the template. $\left.{ }^{[60]} \mathrm{d}\right)$ Schematics of a two-step sCAPA experiment. ${ }^{[60]}$

e) Heterogeneous colloidal dimers composed of different $1 \mu \mathrm{m}$ polystyrene microspheres produced by SCAPA. ${ }^{[60]}$ All subfigures are adapted and reproduced by permission of The Royal Society of Chemistry. assembly, or sCAPA, which is at the basis for the fabrication of a broad range of multifunctional hybrid colloids. ${ }^{[64]}$

The protocol described above enables the possibility to decouple the geometry of the local particle assemblies from their composition. The former is in fact solely set by the shape of the traps on the template, which are designed using lithographic techniques, while the latter is instead defined by the filling sequence of the traps. Capillarity is the driving force for the assembly; hence, the method does not require any specificity in the chemistry of the assembled particles, provided that they can be dispersed in the aqueous droplet and that they can successfully create an accumulation zone. A broad range of materials can therefore be combined into heterogeneous dumbbells, as shown in Fig. 3.

An additional, unique strength of sCAPA is the possibility to apply the same ideas to larger particle numbers and more complex trap geometries, opening up true programmability of the produced patterns. Fig. 4 displays various examples of complex hybrid colloids including 'barcoded' linear particle sequences, triangular,
L-shaped and rectangular colloidal clusters of desired composition. Sequential filling of non-linear traps is possible by appropriately rotating the template with respect to the direction of the moving meniscus, thus making it possible to pack the different particles against a specific side of the assembly template. ${ }^{[64,65]}$

Particles deposited by sCAPA can then be permanently linked and released from the substrate, turning the method from a robust patterning tool into a powerful 'synthesis strategy' for hybrid colloids. We have developed a particularly simple protocol using polymeric particles. These can in fact be easily used as linkers by subjecting the whole template to a gentle heat treatment ( $75^{\circ} \mathrm{C}$ for $10 \mathrm{~min}$ ) that partly melts the surface of polymeric particles and sinters them to their neighbors (see Fig. 5a,b). After sintering, a simple harvesting step is carried out by depositing a small water droplet over the region of interest on the template and freezing it. The ice encapsulates the sintered hybrid colloids, which stay embedded in the frozen droplet after retrieving it from the surface. Upon melting, the particles are directly dispersed in water.

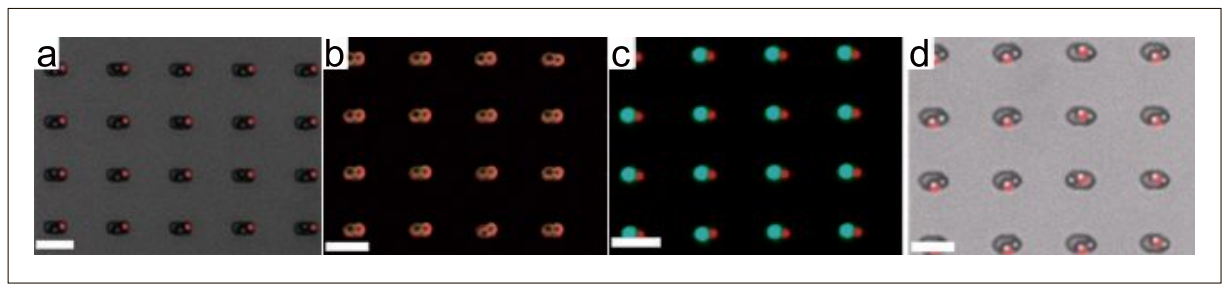

Fig. 3. Heterogeneous dumbbells obtained by sCAPA. ${ }^{[65]}$ a) Polystyrene $/ \mathrm{SiO}_{2}$. b) Polystyrene/ $/ \mathrm{TiO}_{2}$. c) Polystyrene/aminated polystyrene. d) Polystyrene/ $\mathrm{SiO}_{2}$ with $\mathrm{Fe}_{3} \mathrm{O}_{4}$-embedded nanoparticles. The micrographs are overlays of bright-field, dark-field and fluorescence microscopy images. All the particles have a $1 \mu \mathrm{m}$ diameter. Scale bars are $5 \mu \mathrm{m}$. Adapted and reproduced with permission from The Royal Society of Chemistry. 

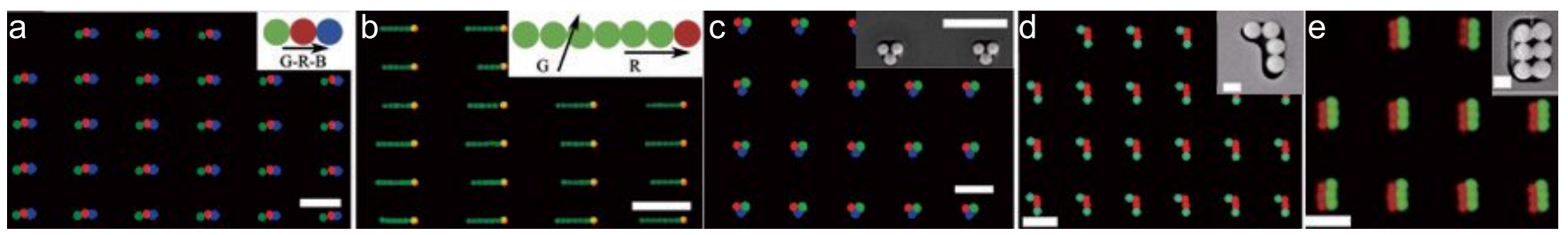

Fig. 4. Different examples of hybrid colloids fabricated by sCAPA. a) A-B-C linear sequences of three different particles. ${ }^{[64]}$ Adapted and reproduced by permission of AAAS Science Advances, copyright 2016. b) 'Colloidal surfactants' with a head particle and a tail of identical particles. ${ }^{[64]}$ Adapted and reproduced by permission of AAAS Science Advances, copyright 2016. c) A-B-C triangular clusters. ${ }^{[64]}$ Adapted and reproduced by permission of AAAS Science Advances, copyright 2016. d) 'L-shaped' clusters with controlled composition both in the short and long arms of the 'L'. ${ }^{[65]}$ Adapted and reproduced with permission from The Royal Society of Chemistry. e) 'Six-pack' rectangular clusters. ${ }^{[65]}$ Adapted and reproduced with permission from The Royal Society of Chemistry. All particles are $1 \mu \mathrm{m}$ diameter polystyrene. The solid arrows in a-b) indicate the deposition directions. All scale bars are $5 \mu \mathrm{m}$, apart from the ones in the insets of $d$-e), which are $1 \mu \mathrm{m}$.

\section{Hybrid Colloids as Versatile Microswimmers}

The possibility of tuning the compositional and geometrical asymmetry of our hybrid clusters makes sCAPA an ideal tool for the fabrication of a broad library of artificial microswimmers. Using this method, we have the possibility to design hybrid colloids whose asymmetry defines the way in which they are going to self-propel. In particular, we have produced a library of hybrid colloids with asymmetries in their dielectric properties that respond to uniform AC electric fields. Existing work has demonstrated that by placing a colloidal particle in the proximity of a conductive surface and by applying $\sim 1 \mathrm{kHz}$ AC electric fields perpendicular to the electrode, the distortion of the local fields produces electro-hydrodynamic flows of ions in solution around the particle. ${ }^{[56,66]}$ In the case of a homogeneous sphere, these flows are symmetric with respect to the center of the particle. Conversely, for asymmetric systems, e.g. dumbbells with differently sized lobes or made of two spheres of different materials, the electro-hydrodynamic flow loses its symmetry and causes propulsion due to viscous drag. We have realized dumbbells with a broad range of compositions, which translate with different speed under the same electric fields depend-

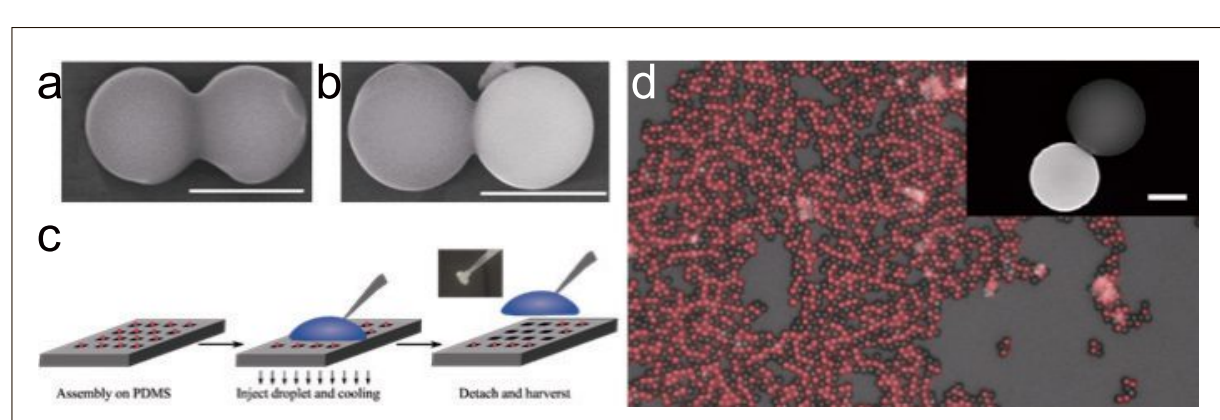

Fig. 5. (a) SEM images of sintered dumbbells consisting of two polystyrene particles and (b) one polystyrene and one silica particle. ${ }^{[64]}$ Adapted and reproduced by permission of AAAS Science Advances, copyright 2016. c) Schematics of the harvesting protocol. d) Heterogeneous polystyrene-silica dumbbells harvested, dispersed in water and dried on a substrate. Inset: SEM image of the same sintered dumbbells. ${ }^{[64]}$ Adapted and reproduced by permission of AAAS Science Advances, copyright 2016. Scale bars are $1 \mu \mathrm{m}$ ing on the materials of the lobes (e.g. the ones in Fig. 3). It is also possible to tune independently the propulsion velocity and the persistency, i.e. the tendency to swim along straight paths, of active trajectories by changing the geometry of the hybrid active colloids. In fact, dimers, square clusters and 'six-pack' rectangular clusters (see Fig. 6a-c) propel under the same conditions with a velocity that only varies between 10 and $15 \mu \mathrm{m} / \mathrm{s}$. They nonetheless show differences in their persistency of more than one order of magnitude, from a few microns for the dumbbells up to 100 $\mu \mathrm{m}$ for the 'six-pack' clusters, which are then able to swim straight for distances corresponding to tens of times their own size. Also, the design flexibility has made it possible to realize clusters whose shape determines their mode of motion. Zig-zag clusters are designed to rotate on the spot. They are constituted by two dumbbells that try to propel along a straight line but are joined off-axis, giving rise to a torque and thus a continuous rotation (Fig. 6d). Finally, 'U-shaped' clusters are fabricated to propel in a straight fashion using two dumbbells but also have the capability to collect and transport cargo particles that they pick up during their motion due to the middle gap. The cargo can then be released on demand by switching off the AC field (see Fig. 6e).

\section{Conclusions and Perspectives}

The examples reported above illustrate how sCAPA, by virtue of decoupling the processes that define the shape and the composition of colloidal clusters, allows the existing bottlenecks in the fabrication of fully programmable hybrid colloids to be overcome. Our approach has significant appeal for the realization of complex building blocks for colloidal assembly, in the spirit of creating colloidal molecules with specific interaction symmetries. Nonetheless, this particle fabrication scheme is a surface-based method and its yield is inherently determined by the density of the traps on the template, by the template size, by the deposition speed and by the reliability of the sequential filling. Even though all of these aspects can be further optimized, the quantities of particles remain unavoidably limited compared to bulk synthetic methods. Programmability is paid at the price of a reduced yield. Currently, $10^{6}$ particles with a very high fidelity, e.g. up to $96 \%$ for dumbbells, can be achieved and up to a 100 -fold increase in the particle number is envisaged by further process optimization (e.g. larger and denser templates, continuous feed of the particle suspension by microfluidic assembly heads $\left.{ }^{[67]}\right)$. These numbers make particles produced by sCAPA of interest for fundamental studies of particle assembly under confinement in 3D, e.g. in small reaction chambers or emulsion droplets, and in 2D, e.g. at fluid interfaces. While, at present such versatile colloids may not yet be useful for the assembly of macroscopic 3D materials, one should nonetheless not think of them only as building blocks for materials. Instead, this method offers the opportunity to realize microscale objects that cannot otherwise be made, where the single hybrid colloid itself can be considered as a small device. The opportunity to design predefined asymmetries leading to active motion, for instance, enables us to envision a new generation of active hybrid colloids, where the incorporation of different materials allows for using and combin- 

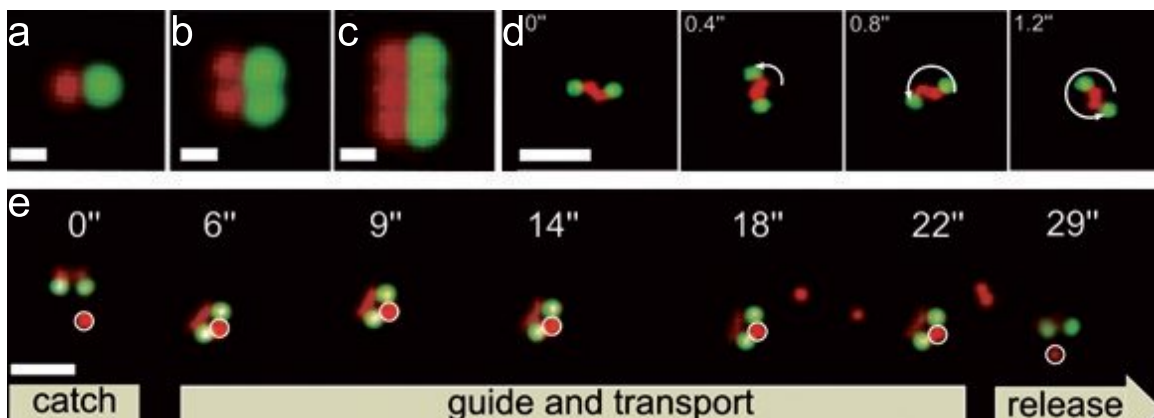

$14^{\prime \prime}$

guide and transport
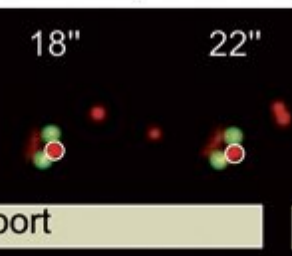

$29^{\prime \prime}$

Fig. 6. (a) Heterogeneous dimers, (b) square clusters and (c) 'six-pack' rectangular clusters propel with similar speed, but with very different persistency. d) Zig-zag clusters instead show continuous rotation on the spot. e) 'U-shaped' clusters can be designed to pick up, transport and release simple cargoes. Scale bars in (a-c) $1 \mu \mathrm{m}$; in (d-e) $5 \mu \mathrm{m}$. The micrographs are fluorescence images and all the particles are $1 \mu \mathrm{m}$ polystyrene spheres with different surface functionalization. ${ }^{[65]}$ All subfigures adapted and reproduced with permission from The Royal Society of Chemistry.

ing different propulsion and guidance strategies, beyond the AC electric field actuation described above as an example. This goal has been recently pursued by numerical models of active microparticles with complex geometries and modes of propulsion, ${ }^{[68,69]}$ and our work demonstrates that experimental analogues can be made.

In summary, we are convinced that the approach briefly reviewed here will become a powerful tool for the production of fully programmable microscale materials, and we envision the combination of many more kinds of particles in the future to produce versatile microscale devices with a broad range of functionalities. Finally, the miniaturization of the methodology to nanoscale systems also carries significant potential for applications in plasmonics, sensing and delivery, where programmable hybrid nanoparticles remain a significant challenge.

\section{Acknowledgements}

We thank Emanuele Marini and Jessica Leemann for their work on the synthesis and characterization of colloidal molecules produced by sCAPA. LI, SN and IB acknowledge financial support from the Swiss National Science Foundation (grant PP00P2_144646/1).

Received: April 10, 2017

[1] S. Suresh, Nat. Mater. 2006, 5, 253.

[2] W. Poon, Science 2004, 304, 830.

[3] P. N. Pusey, in 'Colloidal Suspension', 1991, Vol. 51.

[4] J. Perrin, C. R. Acad. Sci., Paris 1908, 146, 967.

[5] M. D. Haw, J. Phys.: Cond. Mat. 2002, 14, 7769.

[6] T. Bohlein, J. Mikhael, C. Bechinger, Nat. Mater. 2012, 11, 126.

[7] P. J. Yunker, K. Chen, Z. Zhang, A. G. Yodh, Phys. Rev. Lett. 2011, 106, 225503.

[8] S. Deutschländer, T. Horn, H. Löwen, G. Maret, P. Keim, Phys. Rev. Lett. 2013, 111, 098301.

[9] F. Sciortino, Nat. Mater. 2002, 1, 145.

[10] E. Zaccarelli, J. Phys.: Cond. Mat. 2007, 19 , 323101.
[11] D. G. Aarts, M. Schmidt, H. N. Lekkerkerker, Science 2004, 304, 847.

[12] M. E. Leunissen, C. G. Christova, A.-P. Hynninen, C. P. Royall, A. I. Campbell, A. Imhof, M. Dijkstra, R. van Roij, A. van Blaaderen, Nature 2005, 437, 235.

[13] S. C. Glotzer, M. J. Solomon, Nat. Mater. 2007, 6, 557.

[14] L. Cademartiri, K. J. M. Bishop, Nat. Mater. 2015, $14,2$.

[15] F. Li, D. P. Josephson, A. Stein, Angew. Chem. Int. Ed. 2011, 50, 360.

[16] A. Perro, E. Duguet, O. Lambert, J.-C. Taveau, E. Bourgeat-Lami, S. Ravaine, Angew. Chem. Int. Ed. 2009, 48, 361.

[17] D. J. Kraft, W. S. Vlug, C. M. van Kats, A. van Blaaderen, A. Imhof, W. K. Kegel, J. Am. Chem. Soc. 2009, 131, 1182.

[18] Y. Wang, Y. Wang, D. R. Breed, V. N. Manoharan, L. Feng, A. D. Hollingsworth, M. Weck, D. J. Pine, Nature 2012, 491, 51.

[19] V. Meester, R. W. Verweij, C. van der Wel, D. J. Kraft, ACS Nano 2016, 10, 4322.

[20] R. W. Perry, V. N. Manoharan, Soft Matter 2016, 12, 2868.

[21] L. Hong, A. Cacciuto, E. Luijten, S. Granick, Nano Lett. 2006, 6, 2510.

[22] S. K. Smoukov, S. Gangwal, M. Marquez, O. D. Velev, Soft Matter 2009, 5, 1285.

[23] C. Yu, J. Zhang, S. Granick, Angew. Chem. Int. Ed. 2014, 53, 4364.

[24] Q. Chen, S. C. Bae, S. Granick, Nature 2011, $469,381$.

[25] E. Ducrot, M. He, G.-R. Yi, D. J. Pine, Nat. Mater. 2017, advance online publication.

[26] J. J. Crassous, A. M. Mihut, E. Wernersson, P. Pfleiderer, J. Vermant, P. Linse, P. Schurtenberger, Nat. Commun. 2014, 5, 5516.

[27] A. A. Shah, B. Schultz, W. Zhang, S. C. Glotzer, M. J. Solomon, Nat. Mater. 2015, 14, 117.

[28] A. Klinkova, H. Thérien-Aubin, R. M. Choueiri, M. Rubinstein, E. Kumacheva, Proc. Natl. Acad. Sci. USA 2013, 110, 18775.

[29] K. Liu, A. Lukach, K. Sugikawa, S. Chung, J. Vickery, H. Therien-Aubin, B. Yang, M. Rubinstein, E. Kumacheva, Angew. Chem. Int. Ed. 2014, 53, 2648.

[30] A. H. Groschel, A. Walther, T. I. Lobling, F. H. Schacher, H. Schmalz, A. H. E. Muller, Nature 2013, 503, 247.

[31] E. Bianchi, R. Blaak, C. N. Likos, Phys. Chem. Chem. Phys. 2011, 13, 6397.

[32] S. Jiang, Q. Chen, M. Tripathy, E. Luijten, K. S. Schweizer, S. Granick, Adv. Mater. 2010, 22 , 1060.

[33] M. Lattuada, T. A. Hatton, Nano Today 2011, 6, 286.
[34] G.-R. Yi, D. J. Pine, S. Sacanna, J. Phys.: Cond. Matt. 2013, 25, 193101.

[35] A. B. Pawar, I. Kretzschmar, Langmuir 2008, 24,355 .

[36] Z. Nie, W. Li, M. Seo, S. Xu, E. Kumacheva, J. Am. Chem. Soc. 2006, 128, 9408.

[37] S. Berger, A. Synytska, L. Ionov, K.-J. Eichhorn, M. Stamm, Macromolecules 2008, 41, 9669.

[38] S. Sacanna, L. Rossi, D. J. Pine, J. Am. Chem. Soc. 2012, 134, 6112.

[39] J.-G. Park, J. D. Forster, E. R. Dufresne, J. Am Chem. Soc. 2010, 132, 5960.

[40] D. J. Kraft, J. Hilhorst, M. A. P. Heinen, M. J. Hoogenraad, B. Luigjes, W. K. Kegel, J. Phys. Chem. B 2011, 115, 7175 .

[41] V. N. Manoharan, M. T. Elsesser, D. J. Pine, Science 2003, 301, 483.

[42] G. R. Yi, V. N. Manoharan, E. Michel, M. T. Elsesser, S. M. Yang, D. J. Pine, Adv. Mater. 2004, 16, 1204.

[43] A. B. Pawar, I. Kretzschmar, Macromol. Rapid Commun. 2010, 31, 150.

[44] N. Prasad, J. Perumal, C.-H. Choi, C.-S. Lee, D.-P. Kim, Adv. Funct. Mater. 2009, 19, 1656.

[45] Z. Nie, S. Xu, M. Seo, P. C. Lewis, E. Kumacheva, J. Am. Chem. Soc. 2005, 127, 8058.

[46] W. Poon, Phys. Complex Coll. 2013, 184, 317.

[47] S. J. Ebbens, J. R. Howse, Soft Matter 2010, 6 , 726.

[48] C. Bechinger, R. Di Leonardo, H. Löwen, C. Reichhardt, G. Volpe, G. Volpe, Rev. Mod. Phys. 2016, $88,045006$.

[49] W. F. Paxton, K. C. Kistler, C. C. Olmeda, A Sen, S. K. St. Angelo, Y. Cao, T. E. Mallouk, P. E. Lammert, V. H. Crespi, J. Amer. Chem. Soc. 2004, 126, 13424.

[50] J. R. Howse, R. A. Jones, A. J. Ryan, T. Gough, R. Vafabakhsh, R. Golestanian, Phys. Rev. Lett. 2007, 99, 048102.

[51] S. Sánchez, L. Soler, J. Katuri, Angew. Chem. Int. Ed. 2015, 54, 1414.

[52] A. P. Bregulla, H. Yang, F. Cichos, ACS Nano 2014, 8,6542 .

[53] R. Dreyfus, J. Baudry, M. L. Roper, M. Fermigier, H. A. Stone, J. Bibette, Nature 2005, $437,862$.

[54] L. Zhang, J. J. Abbott, L. Dong, B. E. Kratochvil, D. Bell, B. J. Nelson, Appl. Phys. Lett. 2009, 94 , 064107.

[55] A. Ghosh, P. Fischer, Nano Lett. 2009, 9, 2243.

[56] F. Ma, X. Yang, H. Zhao, N. Wu, Phys. Rev. Lett. 2015, 115, 208302.

[57] S. Gangwal, O. J. Cayre, M. Z. Bazant, O. D. Velev, Phys. Rev. Lett. 2008, 100, 058302.

[58] R. D. Deegan, O. Bakajin, T. F. Dupont, G Huber, S. R. Nagel, T. A. Witten, Nature 1997, 389, 827.

[59] P. J. Yunker, T. Still, M. A. Lohr, A. G. Yodh, Nature 2011, 476, 308.

[60] S. Ni, J. Leemann, H. Wolf, L. Isa, L., Faraday Disc. 2015, 181, 225.

[61] Y. Xia, Y. Yin, Y. Lu, J. McLellan, Adv. Funct. Mater. 2003, 13, 907.

[62] T. Kraus, L. Malaquin, H. Schmid, W. Riess, N. D. Spencer, H. Wolf, Nat. Nanotechnol. 2007, 2,570 .

[63] L. Malaquin, T. Kraus, H. Schmid, E. Delamarche, H. Wolf, Langmuir 2007, 23, 11513.

[64] S. Ni, J. Leemann, I. Buttinoni, L. Isa, H. Wolf, Sci. Adv. 2016, 2, e1501779.

[65] S. Ni, E. Marini, I. Buttinoni, H. Wolf, L. Isa Soft Matter, 2017, doi:10.1039/c7sm00443e.

[66] W. D. Ristenpart, I. A. Aksay, D. A. Saville, $J$. Fluid Mech. 2007, 575, 83.

[67] S. Ni, M. J. K. Klein, N. D. Spencer, H. Wolf, Langmuir 2014, 30, 90.

[68] R. Soto, R. Golestanian, Phys.Rev. E 2015, 91, 052304.

[69] R. Soto, R. Golestanian, Phys. Rev. Lett. 2014, 112, 068301. 